\title{
PFA AND $\omega_{1}$-FREE COMPACT SPACES
}

\author{
ALAN DOW AND KLAAS PIETER HART
}

\begin{abstract}
The Proper Forcing Axiom implies that compact Hausdorff spaces are either first-countable or contain a converging $\omega_{1}$-sequence.
\end{abstract}

\section{INTRODUCTION}

This paper continues the investigation from $[5$ into a conjectured dichotomy for compact Hausdorff spaces: every such space is either first-countable or it has a nontrivial converging $\omega_{1}$-sequence. Whether this dichotomy holds was asked by Juhász and a consistent counterexample to this conjecture was given by him, Koszmider, and Soukup in 8 .

The main result in [5] states that the dichotomy holds in a large class of ccc forcing extensions of models in which the Continuum Hypothesis holds. This result does not encompass all ccc extensions and one can rightfully ask what the status of the dichotomy is under Martin's Axiom.

In Section 2 we point out that a space constructed by the first author in 4 witnesses that the combination $M A+\mathfrak{c}=\aleph_{2}$ is not strong enough to imply the dichotomy.

Our main result appears in Section 3, the Proper Forcing Axiom implies the dichotomy. This then also implies that the dichotomy is consistent with and independent of MA $+\mathfrak{c}=\aleph_{2}$. It also improves upon the first author's result in $[3$, Theorem 5.1], which implies that, in turn, PFA implies that a compact space without convergent $\omega_{1}$-sequences must be Fréchet-Urysohn.

\section{Some PRELIMINARIES}

Here we collect the notions that will be used throughout the paper. Others will be defined when needed.

Converging $\omega_{1}$-sequences. The central notion is that of a converging $\omega_{1}$-sequence. An $\omega_{1}$-sequence $\left\langle x_{\alpha}: \alpha \in \omega_{1}\right\rangle$ converges to a point $x$ if for every neighbourhood $U$ of $x$ there is an $\alpha$ such that $\left\{x_{\beta}: \beta \geqslant \alpha\right\} \subseteq U$. Unless stated otherwise our converging sequences are assumed to be non-trivial, that is, injective. A space that contains no non-trivial converging $\omega_{1}$-sequences will be called $\omega_{1}$-free.

In all cases where we construct converging $\omega_{1}$-sequences we use the regularity of the space to show convergence: an $\omega_{1}$-sequence $\left\langle x_{\alpha}: \alpha \in \omega_{1}\right\rangle$ converges to a point $x$ if and only if for every neighbourhood $U$ of $x$ there is an $\alpha$ such that $\left\{x_{\beta}: \beta \geqslant \alpha\right\} \subseteq \operatorname{cl} U$.

We should record here that the dichotomy is indeed a dichotomy. If a point has a countable local base then it is not the limit of a non-trivial $\omega_{1}$-sequence: that sequence would have to be constant on a tail.

Date: Monday 25-10-2021 at 17:48:05 (cest).

2020 Mathematics Subject Classification. Primary: 54D30. Secondary: 03E35, 03E50, 03E57, 54A20, 54A35, 54D20.

Key words and phrases. PFA, compact, convergence, $\omega_{1}$-sequence, first-countable. 
Free sequences. At one point we shall need to know that a compact $\omega_{1}$-free space has countable tightness, which for the purposes of this paper is best defined by a characterization: there is no free $\omega_{1}$-sequence.

An $\omega_{1}$-sequence $\left\langle x_{\alpha}: \alpha \in \omega_{1}\right\rangle$ is free if for every $\alpha$ the sets $\left\{x_{\beta}: \beta<\alpha\right\}$ and $\left\{x_{\beta}: \beta \geqslant \alpha\right\}$ have disjoint closures.

\section{MA IS NOT ENOUGH}

In this section we show that the conjuction of MA and the equality $\mathfrak{c}=\aleph_{2}$ is not strong enough to give a positive answer to Juhász' question. For this we recall the definition of initial $\omega_{1}$-compactness: every open cover of cardinality at most $\aleph_{1}$ has a finite subcover.

In [4, Corollary 5.5] one finds a model of $\mathrm{MA}+\mathfrak{c}=\aleph_{2}$ in which there is a compact space $X$ that is of countable tightness, and has a proper dense subspace $Y$ that is first-countable and initially $\omega_{1}$-compact. As $Y$ is a fortiori countably compact the space $X$ is not first-countable at all points that are not in $Y$. In addition every compact subset of $X \backslash Y$ is finite. We shall show that $X$ is $\omega_{1}$-free.

Assume $\left\langle x_{\alpha}: \alpha \in \omega_{1}\right\rangle$ is a converging $\omega_{1}$-sequence with limit $x$. Since, as noted above, a point of first-countability cannot be the limit of a non-trivial $\omega_{1}$-sequence we must have $x \in X \backslash Y$.

Since $x$ is the only complete accumulation point of the set $\left\{x_{\alpha}: \alpha \in \omega_{1}\right\}$ the initial $\omega_{1}$-compactness of $Y$ implies that all but countably many $x_{\alpha}$ are in $X \backslash Y$.

Thus we assume the $x_{\alpha}$ are all in $X \backslash Y$. For each limit ordinal $\delta$ we choose a cofinal subset $C_{\delta}$ of order type $\omega$ and an accumulation point $y_{\delta}$ of $\left\{x_{\alpha}: \alpha \in C_{\delta}\right\}$; by the assumption on $X \backslash Y$ we can take $y_{\delta}$ in $Y$.

There is a point $z$ in $Y$ with the property that for every neighbourhood $U$ of $z$ the set $\left\{\delta: y_{\delta} \in U\right\}$ is uncountable. But then so is $\left\{\alpha: x_{\alpha} \in U\right\}$ for every such neighbourhood. But then $z=x$ and we have our contradiction.

\section{The MAIN RESUlT}

In this section we prove our main result.

Theorem 1 (PFA). Every compact Hausdorff space either contains a non-trivial converging $\omega_{1}$-sequence or is first-countable.

We shall prove the theorem in a number of steps. We assume PFA and we let $X$ be a compact Hausdorff space that is non first-countable and also $\omega_{1}$-free, and we intend to reach a contradiction by the end of this section.

Lemma 2. Our space $X$ has countable tightness.

Proof. This is established by Juhász and Szentmiklóssy in [9]: a compact space of uncountable tightness contains (even) a free $\omega_{1}$-sequence that converges.

Lemma 3. We may assume, without loss of generality, that $X$ is separable.

Proof. This was established by the present authors in [5]: a compact $\omega_{1}$-free space that is not first-countable contains a closed separable subspace that is not firstcountable.

Henceforth we add separability to the assumptions on our space $X$.

Lemma 4. Our space $X$ has cardinality at most $\mathfrak{c}$.

Proof. In 1] Balogh proved that PFA implies all compact Hausdorff spaces of countable tightness are in fact sequential.

Now apply the elementary fact that the sequential closure of a countable set has cardinality at most $\mathfrak{c}$. 
Our next step is to investigate what happens to our space $X$ when we force with the partial order $\operatorname{Fn}\left(\omega_{1}, 2, \aleph_{1}\right)$ : the set of countable partial functions from $\omega_{1}$ to 2 (notation as in [10]).

The reason for this is that the main steps towards the contradiction involve proper partial orders that are built in two stages, the first of which is the aforementioned partial order $\operatorname{Fn}\left(\omega_{1}, 2, \aleph_{1}\right)$; this partial order is proper and forces $\mathrm{CH}$ to hold.

Lemma 5. Upon forcing with $\operatorname{Fn}\left(\omega_{1}, 2, \aleph_{1}\right)$ our space $X$ remains compact.

Proof. By "our space $X$ remains compact" we mean that the set $X$, with the topology generated by the ground-model open sets, is a compact space.

To begin: our space $X$ does remain countably compact. This is so because every countably infinite subset of $X$ belongs to the ground model and hence has an accumulation point there. Because the old sets form a base for the new topology that point remains an accumulation point.

Now, if $X$ is no longer compact in this extension then [2, Proposition 5.8] applies and there is a proper partial order $\mathbb{P}$ that introduces an embedding $e$ of the ordinal space $\omega_{1}$ into $X$. We shall specify $\aleph_{1}$ many dense sets in the partial order $\operatorname{Fn}\left(\omega_{1}, 2, \aleph_{1}\right) * \dot{\mathbb{P}}$ to determine a map $f: \omega_{1} \rightarrow X$, in our ground model, that is a free sequence in $X$.

First we take, for each $\alpha$, the set $E_{\alpha}=\{p:(\exists x \in X)(p \Vdash \dot{e}(\alpha)=x)\}$. An application of PFA to this family yields a map $f: \omega_{1} \rightarrow X$.

Next we note that in the extension the set $e[\alpha+1]$ is compact and does not meet the closure of $e\left[\left[\alpha+1, \omega_{1}\right)\right]$. Therefore there is an open set $U_{\alpha}$ in the ground model topology with the property that $e[\alpha+1] \subseteq U_{\alpha}$ and $\operatorname{cl} U_{\alpha} \cap e\left[\left[\alpha+1, \omega_{1}\right)\right]=\emptyset$. As above we get dense sets $D_{\alpha}$ that determine the sequence $\left\langle U_{\alpha}: \alpha \in \omega_{1}\right\rangle$.

Now apply PFA to the union of the families to get the desired free sequence $f: \omega_{1} \rightarrow X$.

This contradicts the countable tightness of $X$ and establishes the lemma.

If we combine this with Lemma 4 then we know that in the extension by $\operatorname{Fn}\left(\omega_{1}, 2, \aleph_{1}\right)$ our space $X$ has become a compact space of cardinality and weight at most $\aleph_{1}$. This will be used in the next step.

From [5] we quote (and modify) the notion ultra-Fréchet, a useful technical property that will be used in our proof.

Definition 6. A space $Z$ will be said to be ultra-Fréchet if it has countable tightness and for each countable subset $D$ of $Z$ and each free ultrafilter $\mathcal{U}$ on $D$ that converges in $Z$ there is a countable subfamily $\mathcal{U}^{\prime}$ of $\mathcal{U}$ with the property that every infinite pseudointersection of $\mathcal{U}^{\prime}$ converges.

This is a weakening of the original definition, which did not require that the ultrafilter $\mathcal{U}$ be convergent and thus would imply that the space is countably compact. In the present context of compact spaces there is no difference but the notion may be useful outside this class.

We also remark that all pseudointersections of the countable subfamily converge to the same point (the union of two pseudointersections is again a pseudointersection), to wit the limit of the ultrafilter.

Lemma 7. Our space $X$ is ultra-Fréchet, also upon forcing with $\operatorname{Fn}\left(\omega_{1}, 2, \aleph_{1}\right)$.

Proof. We argue by contradiction and assume that in the extension there are a countable set $D$ and an ultrafilter $\mathcal{U}$ on $D$ where the property fails; that is: every countable subfamily of $\mathcal{U}$ has an infinite pseudointersection that does not converge. 
This will also cover the ground model case: because the forcing adds no new countable sets a 'bad' pair $\langle D, \mathcal{U}\rangle$ from the ground model is still 'bad' in the extension.

We note that by sequentiality of $X$ every countably infinite subset of $X$ contains an infinite subset that converges: if the set is closed then it is compact metrizable and hence contains a convergent sequence, and if it is not closed then sequentiality gives us a sequence in the set that converges to a point outside the set.

We now construct, recursively, an almost disjoint family $\left\{a_{\alpha}: \alpha \in \omega_{1}\right\}$ of infinite subsets of $D$ that converge, say $a_{\alpha}$ converges to $y_{\alpha}$, where $y_{\alpha} \neq x$. Note that none of the $a_{\alpha} \mathrm{s}$ can belong to $\mathcal{U}$ : the countable, indeed singleton, family $\left\{a_{\alpha}\right\}$ would contradict our assumption. We also choose, for each $\alpha$, a neighbourhood $O_{\alpha}$ of $x$ such that $y_{\alpha} \notin \operatorname{cl} O_{\alpha}$.

We enumerate $\mathcal{U}$ as $\left\langle U_{\alpha}: \alpha \in \omega_{1}\right\rangle$.

At stage $\alpha$ we let $c_{\alpha}$ be an infinite pseudointersection of $\left\{U_{\beta}: \beta<\alpha\right\} \cup\left\{D \cap O_{\beta}\right.$ : $\beta<\alpha\}$ that does not converge.

As noted above $c_{\alpha}$ has an infinite subset that converges; if every infinite convergent subset of $c_{\alpha}$ were to converge to $x$ then by sequentiality the sequential closure of $c_{\alpha}$ would be just $c_{\alpha} \cup\{x\}$, and $c_{\alpha}$ would converge after all. So there is an infinite convergent subset $a_{\alpha}$ of $c_{\alpha}$ that converges to some point $y_{\alpha}$ distinct from $x$.

The family $\left\{a_{\alpha}: \alpha \in \omega_{1}\right\}$ and the ultrafilter $\mathcal{U}$ satisfy the conditions of 6 , Lemma 1.8]. Therefore there is a ccc partial order $\mathbb{Q}$ that introduces an uncountable subset $I$ of $\omega_{1}$ such that $\left\{a_{\alpha}: \alpha \in I\right\}$ is a Luzin family. This means that for every $\alpha \in I$ and every natural number $n$ the set $F(\alpha, n)=\left\{\beta \in I \cap \alpha: a_{\beta} \cap a_{\alpha} \subseteq n\right\}$ is finite.

It takes only $\aleph_{1}$ many dense sets in the partial order $\operatorname{Fn}\left(\omega_{1}, 2, \aleph_{1}\right) * \dot{\mathbb{Q}}$ to determine such an uncountable set $I$, the function $\alpha \mapsto a_{\alpha} \cup\left\{y_{\alpha}\right\}$ and the finite sets $F(\alpha, n)$. Therefore an application of PFA yields the existence of such sets in the ground model, and we may as well assume $I=\omega_{1}$.

The sequence $\left\langle y_{\alpha}: \alpha \in \omega_{1}\right\rangle$ converges, in the ground model. Indeed, let $K=$ $\bigcap_{\alpha} \operatorname{cl}\left\{y_{\beta}: \beta \geqslant \alpha\right\}$. By compactness $K$ is non-empty of course and by the Luzin property of the family $\left\{a_{\alpha}: \alpha \in \omega_{1}\right\}$ it consists of just one point and $\left\langle y_{\alpha}: \alpha \in \omega_{1}\right\rangle$ converges to that point, which contradicts our standing assumption on $X$.

To see this, let $x \in K$ and let $O$ be an arbitrary neighbourhood of $x$. The set $A=\left\{\alpha: y_{\alpha} \in O\right\}$ is uncountable, and for each $\alpha \in A$ there is an $m_{\alpha} \in \omega$ such that $a_{\alpha} \backslash O \subseteq m_{\alpha}$. We may assume that there is a single $m$ such that $m_{\alpha}=m$ for all $\alpha \in A$.

We claim that $\left\{\beta: y_{\beta} \notin \mathrm{cl} O\right\}$ is countable; by regularity of $X$ this suffices to prove that $\left\langle y_{\alpha}: \alpha \in \omega_{1}\right\rangle$ converges to $x$.

If the set is uncountable then, by the same argument as above, we find an uncountable set $B$ and an $n \geqslant m$ such that $a_{\beta} \cap O \subseteq n$ for all $\beta \in B$. But now take $\alpha \in A$ such that $\alpha \cap B$ is infinite. Then $a_{\beta} \cap a_{\alpha} \subseteq n$ for all $\beta \in \alpha \cap B$, contradicting the Luzin property of our family.

We can now take the last step towards our contradiction. To recapitulate: we assume our space $X$ is compact, separable, not first-countable and $\omega_{1}$-free. From this we deduced that $X$ remains compact after forcing with the partial order $\operatorname{Fn}\left(\omega_{1}, 2, \aleph_{1}\right)$, and that it is ultra-Fréchet before and after this forcing. In addition, its separability and non-first-countability are preserved by $\operatorname{Fn}\left(\omega_{1}, 2, \aleph_{1}\right)$ too.

Lemma 8. The assumptions that $X$ is compact, separable, not first-countable and ultra-Fréchet, before and after forcing with $\operatorname{Fn}\left(\omega_{1}, 2, \aleph_{1}\right)$, imply that $X$ has a converging $\omega_{1}$-sequence. 
Proof. We work in the extension by $\operatorname{Fn}\left(\omega_{1}, 2, \aleph_{1}\right)$ and take a sequence $\left\langle M_{\alpha}: \alpha \in \omega_{1}\right\rangle$ of countable elementary substructures of $H(\kappa)$ for a suitable large cardinal $\kappa$ such that

- $X$ belongs to $M_{0}$

- $M_{\alpha}=\bigcup_{\beta<\alpha} M_{\beta}$ if $\alpha$ is a limit

- $\left\langle M_{\beta}: \beta \leqslant \alpha\right\rangle \in M_{\alpha+1}$ and $M_{\alpha} \prec M_{\alpha+1}$ for all $\alpha$

By elementarity there are $z \in M_{0} \cap X$ and $D \in M_{0}$ such that $X$ is not first-countable at $z$ and $D$ is countable and dense in $X$. We fix such $z$ and $D$.

Elementarity also implies that $M_{\alpha}^{\omega} \in M_{\alpha+1}$ for all $\alpha$ and this, combined with the Continuum Hypothesis, implies that every countable subset of $M=\bigcup_{\alpha<\omega_{1}} M_{\alpha}$ is a member of $M$. In particular $\mathcal{P}(D) \subseteq M$.

At this point we could refer to [5, Proof of Proposition 2.8], which produces sequences $\left\langle a_{\alpha}: \alpha \in \omega_{1}\right\rangle$ and $\left\langle b_{\alpha}: \alpha \in \omega_{1}\right\rangle$ of infinite subsets of $D$ and a sequence $\left\langle y_{\alpha}: \alpha \in \omega_{1}\right\rangle$ of points in $X \backslash\{z\}$ with the following properties:

(1) $a_{\alpha}$ converges to $z$

(2) $b_{\alpha}$ converges to $y_{\alpha}$

(3) $a_{\alpha} \cap b_{\alpha}=\emptyset$

(4) $\left\{A: a_{\alpha} \cup b_{\alpha} \subseteq^{*} A\right\}$ is an ultrafilter in $M_{\alpha} \cap \mathcal{P}(D)$

(5) $a_{\alpha}, b_{\alpha}$, and $y_{\alpha}$ belong to $M_{\alpha+1}$

However, for the benefit of the reader we will give a somewhat more streamlined construction than the one referred to above.

Fix an $\alpha \in \omega_{1}$ and let $y_{\alpha}$ be a point in the intersection

$$
\bigcap\left\{U: U \in M_{\alpha}, z \in U, U \text { is open in } X\right\}
$$

that is distinct from $z$. Such a point exists because $\{z\}$ is not a $G_{\delta}$-set in $X$. Moreover, by elementarity we can assume $y_{\alpha} \in M_{\alpha+1}$.

Next observe that $y_{\alpha} \notin \operatorname{cl} A$ whenever $A \in M_{\alpha} \cap \mathcal{P}(D)$ and $z \notin \operatorname{cl} A$. There is an ultrafilter $\mathcal{U}_{1}$ on the Boolean algebra $M_{\alpha} \cap \mathcal{P}(D)$ that extends the filter dual to $\{A: z \notin \operatorname{cl} A\}$ and is such that $y_{\alpha} \in \bigcap\left\{\operatorname{cl} U: U \in \mathcal{U}_{1}\right\}$; again we can assume $\mathcal{U}_{1} \in M_{\alpha+1}$. By the contrapositive of the first sentence of this paragraph we have $z \in \bigcap\left\{\operatorname{cl} U: U \in \mathcal{U}_{1}\right\}$. Next we take ultrafilters $\mathcal{U}_{2}$ and $\mathcal{U}_{3}$ on $D$ that extend $\mathcal{U}_{1}$ and converge to $z$ and $y_{\alpha}$ respectively, yet again we take these ultrafilters in $M_{\alpha+1}$.

Now apply the ultra-Fréchet property to $\mathcal{U}_{2}$ and $y_{\alpha}$, and to $\mathcal{U}_{3}$ and $z$ to find countable subfamilies $\mathcal{U}_{2}^{\prime}$ and $\mathcal{U}_{3}^{\prime}$ as in the definition, where we may assume that both families contain $\mathcal{U}_{1}$. These choices can be made in $M_{\alpha+1}$ and we can then take pseudointersections $a_{\alpha}$ and $b_{\alpha}$ of $\mathcal{U}_{2}^{\prime}$ and $\mathcal{U}_{3}^{\prime}$ respectively that belong to $M_{\alpha+1}$. By the remark after Definition $6 a_{\alpha}$ converges to $z$ and $b_{\alpha}$ converges to $y_{\alpha}$, and we can assume $a_{\alpha} \cap b_{\alpha}=\emptyset$.

All five conditions are met: we took care of (1), (2), (3), and (5) explicitly; (4) holds because $a_{\alpha}$ and $b_{\alpha}$ are both pseudointersections of $\mathcal{U}_{1}$.

Conditions (4) and (5) imply that the sequences $\left\langle a_{\alpha}: \alpha \in \omega_{1}\right\rangle$ and $\left\langle b_{\alpha}: \alpha \in \omega_{1}\right\rangle$ are not $\sigma$-separated in a strong way: whenever $\mathcal{A}$ is a countable family of subsets of $D$ there is an $\alpha$ such that for every $A \in \mathcal{A}$ either $A \cap\left(a_{\alpha} \cup b_{\alpha}\right)$ is finite or $a_{\alpha} \cup b_{\alpha} \subseteq^{*} A$.

We may then apply a result of Todorčević, see [7, p. 145], that implies there is a proper partial order $\mathbb{T}$ that produces an uncountable subset $J$ of $\omega_{1}$ such that $\left(a_{\alpha} \cap b_{\beta}\right) \cup\left(a_{\beta} \cap b_{\alpha}\right)$ is non-empty whenever $\alpha, \beta \in J$ are distinct.

As before we need $\aleph_{1}$ many dense sets in the proper partial order $\operatorname{Fn}\left(\omega_{1}, 2, \aleph_{1}\right) * \dot{\mathbb{T}}$ to determine the sequences $\left\langle a_{\alpha}: \alpha \in \omega_{1}\right\rangle,\left\langle b_{\alpha}: \alpha \in \omega_{1}\right\rangle$, and $\left\langle y_{\alpha}: \alpha \in \omega_{1}\right\rangle$ as well as the uncountable set $J$. We apply PFA and obtain items with properties (1), (2) 
and (3) in our list above as well as the uncountable set $J$ from Todorčević's result, which we may assume to be $\omega_{1}$ itself.

We claim that $\left\langle y_{\alpha}: \alpha \in \omega_{1}\right\rangle$ converges to $z$, which is the final contradiction that we seek. Let $O$ be a neighbourhood of $z$. Because every $a_{\alpha}$ converges to $z$ we know that $a_{\alpha} \backslash O$ is always finite. We claim that $b_{\alpha} \cap O$ is finite for only countably many $\alpha$, and hence that there is an $\alpha$ such that $y_{\beta} \in \operatorname{cl} O$ for $\beta \geqslant \alpha$.

Indeed, assume $b_{\alpha} \cap O$ is finite for uncountably many $\alpha$ and fix two finite sets $F$ and $G$ such that the set $A$ of those $\alpha$ for which $F=a_{\alpha} \backslash O$ and $G=b_{\alpha} \cap O$ is uncountable. Now let $\alpha, \beta \in A$ be distinct. Then $a_{\alpha}=F \cup\left(a_{\alpha} \cap O\right)=\left(a_{\beta} \backslash O\right) \cup$ $\left(a_{\alpha} \cap O\right)$ and $b_{\beta}=G \cup\left(b_{\beta} \backslash O\right)=\left(b_{\alpha} \cap O\right) \cup\left(b_{\beta} \backslash O\right)$, which implies $a_{\alpha} \cap b_{\beta}=\emptyset$. Likewise $a_{\beta} \cap b_{\alpha}=\emptyset$ and we are done.

\section{REFERENCES}

[1] Zoltán T. Balogh, On compact Hausdorff spaces of countable tightness, Proc. Amer. Math. Soc. 105 (1989), no. 3, 755-764, DOI 10.2307/2046929. MR930252

[2] Alan Dow, An introduction to applications of elementary submodels to topology, Topology Proc. 13 (1988), no. 1, 17-72. MR1031969

[3] _ Set-theoretic update on topology, Recent progress in general topology. III, Atlantis Press, Paris, 2014, pp. 329-357, DOI 10.2991/978-94-6239-024-9_7. MR3205487

[4] _ Generalized side-conditions and Moore-Mrówka, Topology Appl. 197 (2016), 75-101, DOI 10.1016/j.topol.2015.10.016. MR3426909

[5] Alan Dow and Klaas Pieter Hart, Reflecting Lindelöf and converging $\omega_{1}$-sequences, Fund. Math. 224 (2014), no. 3, 205-218, DOI 10.4064/fm224-3-1. MR3194414

[6] Alan Dow and Saharon Shelah, Martin's axiom and separated mad families, Rend. Circ. Mat. Palermo (2) 61 (2012), no. 1, 107-115, DOI 10.1007/s12215-011-0078-7. MR2897749

[7] Ilijas Farah, Analytic quotients: theory of liftings for quotients over analytic ideals on the integers, Memoirs of the American Mathematical Society 148 (2000), no. 702, xvi+177. MR1711328 (2001c:03076)

[8] István Juhász, Piotr Koszmider, and Lajos Soukup, A first countable, initially $\omega_{1}$ compact but non-compact space, Topology Appl. 156 (2009), no. 10, 1863-1879, DOI 10.1016/j.topol.2009.04.004. MR2519221

[9] I. Juhász and Z. Szentmiklóssy, Convergent free sequences in compact spaces, Proceedings of the American Mathematical Society 116 (1992), no. 4, 1153-1160, DOI 10.2307/2159502. MR1137223 (93b:54024)

[10] Kenneth Kunen, Set theory. An introduction to independence proofs, Studies in Logic and the Foundations of Mathematics, vol. 102, North-Holland Publishing Co., Amsterdam, 1980. MR597342 (82f:03001)

Department of Mathematics, UnC-Charlotte, 9201 University City Blvd., CharLOTTE, NC 28223-0001

Email address: adow@uncc.edu

$U R L:$ https://webpages.uncc.edu/adow

Faculty EemCS, TU Delft, Postbus 5031, 2600 GA Delft, the Netherlands

Email address: k.p.hart@tudelft.nl

$U R L:$ http://fa.its.tudelft.nl/ ^hart 